\title{
DESCRIPCIÓN DE LOS EVENTOS DE SEQUÍA METEOROLÓGICA EN LOCALIDADES DE LA CORDILLERA CENTRAL, VENEZUELA
}

\author{
Event description of meteorological drought \\ in locations central mountains, Venezuela
}

\section{Adriana Cortez ${ }^{1}$, Barlin Orlando Olivares ${ }^{2}$, Raquel Mayela PARra $^{3}$, Deyanira Lobo ${ }^{4}$, JuAn C. Rey B ${ }^{1,3}$ y María F. RodríGueZ $^{1}$}

Cómo citar: Cortez, A., Orlando Olivares, B., Mayela Parra, R., Lobo, D., Rey B, J., \& Rodríguez, M. (2018). Descripción de los eventos de sequía meteorológica en localidades de la Cordillera Central, Venezuela. Ciencia, Ingenierías y Aplicaciones, 1(1), 23-45. doi:http://dx.doi.org/10.22206/cyap.2018.v1i1.pp23-45

\section{Resumen}

La sequia representa una de las causas más importantes de malnutrición y hambre. Sus efectos pueden ser minimizados si se conocen las zonas más vulnerables, ante su intensidad y periodicidad, para poder hacerle frente mediante medidas preventivas, mitigantes y correctivas. El objetivo de esta investigación fue describir el comportamiento de la sequía meteorológica en tres localidades de la Cordillera Central de Venezuela, tomando como base la información de precipitación de tres estaciones de la red agrometeorológica del Instituto Nacional de Investigaciones Agrícolas (INIA), mediante el Índice de Precipitación Estandarizada (SPI, Standardized Precipitation Index), haciendo énfasis en el inicio, intensidad, duración, magnitud y fre-

\footnotetext{
1 1INIA. Centro Nacional de Investigaciones Agropecuarias (CENIAP). Laboratorio de sistemas de Información en Recursos Agroecológicos, Aragua, Venezuela. Correo: acortez.inia@gmail.com.

2 Investigador. Doctorando del Programa Iberoamericano de Doctores en Agroalimentación de la Universidad de Córdoba (UCO), España.

3 Universidad Central de Venezuela (UCV). Facultad de Agronomía, Departamento de Ingeniería Agrícola, Aragua, Venezuela.

4 Universidad Central de Venezuela (UCV). Facultad de Agronomía, Departamento de Edafología, Aragua, Venezuela.
} 
cuencia de ocurrencia de la sequía. Las magnitudes máximas más relevantes fueron las registradas en la estación Tapipa-Padrón durante el año 2001 cuya magnitud fue de 9,70 y en 2003 con una magnitud de 9,50; también la estación CENIAP reporto una magnitud máxima de 9,20 durante el año 1982. Los resultados de la estimación del SPI coadyuvarán en la identificación de las áreas vulnerables a la sequía, a partir de lo cual se podrán establecer propuestas para el diseño de estrategias que sirvan al manejo integral del recurso hídrico y contribuyan a minimizar el riesgo para la seguridad agroalimentaria.

Palabras clave: vulnerabilidad agrícola; cambio climático; precipitación; déficit hídrico.

\begin{abstract}
Drought is one of the most important causes of malnutrition and hunger. Its effects can be minimized if the most vulnerable areas are known to their intensity and frequency, in order to cope with preventive, mitigating and corrective measures. The aim of this study was to describe the behavior of meteorological drought in three locations in the Central Cordillera of Venezuela, based on the information of precipitation three stations agrometeorological network of the $\mathrm{Na}$ tional Institute for Agricultural Research (INIA), by Standardized Precipitation Index (SPI), emphasizing the onset, intensity, duration, magnitude and frequency of occurrence of drought. The most relevant maximum magnitudes were recorded in the Tapipa-Padron station in 2001 with a magnitude of 9.70 and in 2003 with a magnitude of 9.50; CENIAP station also reported a maximum magnitude of 9.20 during 1982. The results of the estimation of SPI will contribute in identifying vulnerable to drought areas, from which proposals may be established for the design of strategies serving the integrated management of water resources and help to minimize the risk to the food safety.
\end{abstract}

Keywords: agricultural vulnerability; climate change; precipitation; hydric deficit. 


\section{Introducción}

El comportamiento del clima, sobre todo lo relacionado a la ocurrencia de eventos meteorológicos extremos, reviste vital interés para la vida social del hombre. Sus consecuencias se reflejan en diferentes esferas de la economía, pero muy especialmente, en la agricultura, pues ésta puede ser considerada como una gran fábrica a la intemperie, en la cual todas las actividades que en ella se realizan son dependientes del tiempo atmosférico y del clima (OMM, 2006; Solano et al., 2007).

El Grupo Intergubernamental de Expertos sobre el Cambio Climático (IPCC por sus siglas en Inglés) (2013) establece que en las últimas décadas, en distintas partes del mundo, pareciera que las sequías se manifiestan con mayor frecuencia de ocurrencia, con mayor grado de persistencia y una significativa proporción; además, los modelos climáticos demuestran escenarios aún más severos para el presente siglo. En países de gran extensión, como Brasil, China, India, Estados Unidos de América o Australia, una sequía rara vez llega a afectar a la totalidad del país; sin embargo, en las regiones afectadas en estos países por la ocurrencia de este fenómeno se presentan impactos relevantes en la economía nacional y en la sociedad en general.

En el caso particular de Venezuela, gran parte del territorio fue afectado en el último decenio por la ocurrencia de varias sequías extremas. Entre 1997 y 1998, ocurrió una sequía que redujo un 55\% el caudal aportante a la Central Hidroeléctrica Simón Bolívar (antiguamente, Gurí). De igual manera, durante los años 2001 y 2002 se presentaron eventos de sequía que afectaron fuertemente los embalses de Camatagua y Guárico. Posteriormente, en los años 2003 y 2007 sucedieron temporadas secas muy extremas que mermaron las reservas hídricas en los principales embalses situados en Los Llanos y la región Centro Costera (Hernández, 2008; Olivares, 2017). Recientemente, en al año 2010, ocurrió una sequía tan severa, que el Gobierno Nacional implementó un racionamiento eléctrico en la mayor parte de la nación. 
De acuerdo a las actividades económicas que se desarrollan en la zona central del país, se presenta el carácter vulnerable de esta zona a la ocurrencia de eventos de sequía, por lo cual es de vital importancia tener un mejor entendimiento sobre las variaciones de la precipitación, sus tendencias y escenarios futuros. Bajo la influencia del cambio climático, dichos efectos pueden ser minimizados si se conocen las zonas más susceptibles, ante su intensidad y periodicidad, para hacerle frente mediante medidas preventivas.

La hipótesis de trabajo planteada, considera que la sequía en las localidades de la cordillera central de Venezuela es el resultado del componente climático, que determina el riesgo de que se produzca una anomalía pluviométrica, y su impacto en el componente socioeconómico, que define la fragilidad de la población ante ese riesgo. Por lo tanto, la sequía debe ser cuantificada considerando ambos componentes.

El presente trabajo tiene como objetivo describir el comportamiento de la sequía meteorológica de diferentes zonas de la cordillera central de Venezuela, utilizando el Índice de Precipitación Estandarizada (SPI, Standardized Precipitation Index), precisando el inicio, la intensidad, la duración y magnitud de este fenómeno climático.

\section{Materiales y métodos}

\subsection{Unidad de estudio}

En los valles interiores del tramo central de la Cordillera de la Costa se encuentran las principales ciudades de importancia económica para el país. En esta región se asienta la mayor proporción de población nacional caracterizada por la escasez en las fuentes de agua. Entre los principales problemas ambientales están las elevadas tasas de deforestaciones, la erosión de los suelos debido al uso y manejo de las tierras, incluyendo el excesivo pastoreo. En el caso del estado 
Miranda, una parte de la actividad agrícola subsiste en competencia con el turismo. Estas zonas poseen un clima tropical lluvioso, con una época seca determinada por la Alta Presión del Atlántico y una época de lluvias generada por el avance de la Zona de Convergencia Intertropical.

En esta investigación, la unidad de estudio está constituida por tres estaciones: CENIAP, Tapipa Padrón y Ocumare de la Costa, las cuales conforman la red de estaciones agrometeorológicas del INIA, abarcando la región de los Llanos (estados: Aragua y Miranda) (Figura 1).
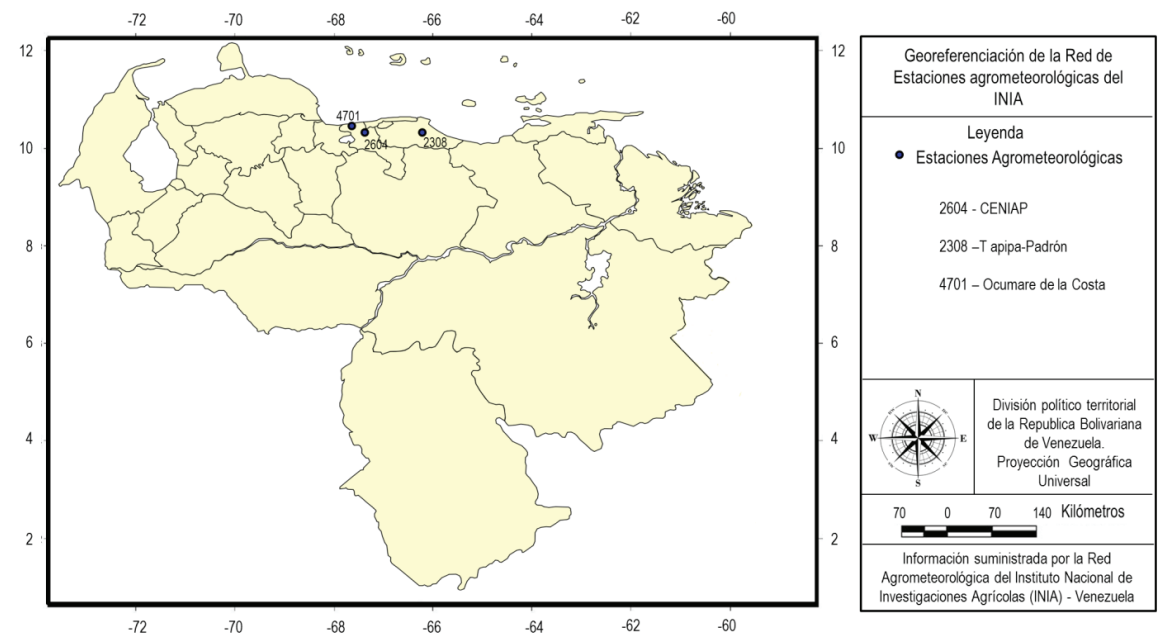

Figura 1. Distribución geográfica de la Red de estaciones agrometeorológicas del INIA en la Cordillera Central Venezolana.

La Tabla 1 presenta la información de la metadata básica representada por el serial nacional, el cual es un código asignado por el organismo responsable de la estación meteorológica, nombre de la estación, fecha de instalación, altitud en m.s.n.m, ubicación en grados, minutos y segundos para obtener la georeferenciación (Latitud y Longitud). Es conveniente señalar que el período de años utilizados en este estudio corresponde al indicado desde la fecha de instalación hasta el último año de registro de cada estación agrometeorológica con la finalidad de conocer la variación temporal de los eventos de 
sequía en el trascurso histórico para esas localidades. La tabla 2 presenta la variabilidad de la precipitación mensual durante el período de estudio en la estación CENIAP.

Tabla 1. Listado de estaciones agrometeorológicas bajo estudio.

\begin{tabular}{lccccccc}
\hline \multicolumn{1}{c}{ Estación } & Estado & Serial & Periodo & Altura m.s.n.m & Latitud & dec. & Longitud \\
\hline CENIAP & Aragua & 2604 & $1980-2014$ & 480 & 10,1714 & $-67,3602$ \\
Tapipa - Padrón & Miranda & 2308 & $1980-2014$ & 38 & 10,1314 & $-66,1757$ \\
Ocumare de la Costa & Aragua & 4701 & $1998-2008$ & 15 & 10,2412 & $-67,4602$ \\
\hline
\end{tabular}

Tabla 2. Serie de datos de precipitación mensual (1980-2014) en la estación CENIAP, Estado Aragua.

\begin{tabular}{|c|c|c|c|c|c|c|c|c|c|c|c|c|c|}
\hline Año & Ene & Feb & Mar & Abr & May & Jun & Jul & Ago & Sep & Oct & Nov & Dic & Anual \\
\hline 1980 & $\mathbf{0 , 0}$ & 0,0 & 0,0 & 31,4 & 131,4 & 115,3 & 138,0 & 159,6 & 187,3 & 152,2 & 15,3 & 11,2 & 941,7 \\
\hline 1981 & 8,2 & 24,1 & 0,0 & 244,3 & 196,2 & 85,8 & 348,6 & 195,3 & 162,3 & 53,2 & 166,2 & 57,6 & 1541,8 \\
\hline 1982 & 3,0 & 1,0 & 0,0 & 205,3 & 138,6 & 30,8 & 38,2 & 134,2 & 124,0 & 165,3 & 0,3 & 1,6 & 842,3 \\
\hline 1983 & 0,0 & 0,0 & 1,0 & 81,9 & 120,7 & 145,6 & 135,9 & 189,8 & 104,0 & 107,7 & 35,7 & 0,8 & 923,1 \\
\hline 1984 & 1,5 & 1,4 & 0,0 & 28,2 & 15,1 & 114,2 & 180,6 & 139,9 & 88,1 & 90,5 & 76,7 & 10,4 & 746,6 \\
\hline 1985 & 0,0 & 0,5 & $\mathbf{0 , 0}$ & 45,5 & 74,9 & 129,9 & 93,3 & 240,3 & 82,9 & 110,5 & 112,3 & 52,6 & 942,7 \\
\hline 1986 & 0,0 & 7,1 & 0,0 & 102,7 & 165,8 & 173,9 & 104,8 & 125,9 & 116,9 & 114,5 & 26,0 & 3,8 & 941,4 \\
\hline 1987 & 3,4 & 0,4 & 9,7 & 46,8 & 169,4 & 167,8 & 99,0 & 136,0 & 169,4 & 109,7 & 70,9 & 0,0 & 982,5 \\
\hline 1988 & 0,0 & 11,5 & 0,0 & 13,0 & 35,1 & 209,9 & 149,0 & 199,8 & 238,5 & 160,7 & 140,1 & 16,6 & 1174,2 \\
\hline 1989 & 3,0 & 0,8 & 0,0 & 2,5 & 142,6 & 48,4 & 79,0 & 243,5 & 219,2 & 136,7 & 78,7 & 1,3 & 955,7 \\
\hline 1990 & 0,0 & 0,6 & 0,0 & 48,5 & 97,3 & 143,5 & 174,2 & 159,8 & 149,7 & 168,3 & 59,0 & 35,8 & 1036,7 \\
\hline 1991 & 0,0 & 0,4 & 12,5 & 104,6 & 45,6 & 47,2 & 186,0 & 262,6 & 103,2 & 64,1 & 77,7 & 14,1 & 918,0 \\
\hline 1992 & 0,0 & 0,0 & 0,0 & 27,4 & 133,7 & 73,8 & 156,7 & 150,6 & 144,0 & 139,9 & 110,4 & 41,5 & 978,0 \\
\hline 1993 & 0,0 & 0,0 & 15,3 & 153,0 & 223,9 & 94,2 & 129,2 & 201,0 & 69,8 & 95,7 & 85,0 & 0,0 & 1067,1 \\
\hline 1994 & 0,0 & 10,7 & 57,7 & 40,2 & 27,6 & 61,6 & 151,1 & 172,3 & 253,2 & 141,8 & 83,0 & 9,1 & 1008,3 \\
\hline 1995 & 3,9 & 0,0 & 147,1 & 16,1 & 35,1 & 65,9 & 269,6 & 141,2 & 232,3 & 117,3 & $\mathbf{5 5 , 7}$ & 4,8 & 1089,0 \\
\hline 1996 & 2,9 & $\mathbf{0 , 0}$ & 7,5 & 19,4 & 116,4 & 213,6 & 282,1 & 217,4 & 235,1 & 176,1 & 68,9 & 82,1 & 1421,5 \\
\hline 1997 & 0,0 & 14,8 & 0,0 & 52,0 & 77,1 & 83,4 & 283,8 & 125,9 & 95,4 & 86,0 & 38,1 & 0,0 & 856,5 \\
\hline 1998 & 0,0 & 0,4 & 38,5 & 136,9 & 202,5 & 107,6 & 101,7 & 189,8 & 116,2 & 131,6 & 17,1 & 5,2 & 1047,5 \\
\hline 1999 & 0,7 & 0,0 & 0,0 & 214,0 & 52,7 & 124,4 & 312,1 & 309,9 & 228,7 & 184,3 & 149,4 & 34,4 & 1610,6 \\
\hline 2000 & 19,5 & 0,0 & 47,6 & 0,0 & 80,8 & 35,6 & 64,3 & 179,1 & 262,3 & 77,8 & 46,2 & 3,0 & 816,2 \\
\hline 2001 & 0,0 & 0,0 & 0,0 & 2,9 & 22,2 & 61,1 & 126,7 & 110,1 & 99,1 & 214,8 & 58,4 & 27,8 & 723,1 \\
\hline 2002 & 1,4 & 0,0 & 1,5 & 66,5 & 165,1 & 127,8 & 67,3 & 128,2 & 139,3 & 72,6 & 37,1 & 0,6 & 807,4 \\
\hline 2003 & 0,0 & 0,0 & 0,0 & 84,1 & 146,0 & 82,7 & 135,9 & 229,3 & 144,0 & 194,3 & 201,3 & 10,8 & 1228,4 \\
\hline 2004 & 0,0 & 0,0 & 0,0 & 77,9 & 172,1 & 82,7 & 122,9 & 170,2 & 177,4 & 107,2 & 87,5 & 17,8 & 1015,7 \\
\hline 2005 & 67,5 & 24,1 & 0,0 & 76,3 & 80,8 & 174,5 & 225,0 & 245,3 & 159,4 & 177,7 & 228,5 & 8,3 & 1467,4 \\
\hline
\end{tabular}




\begin{tabular}{|c|c|c|c|c|c|c|c|c|c|c|c|c|c|}
\hline 2006 & 11,0 & 0,0 & 11,5 & 0,9 & 123,8 & 143,0 & 91,6 & 210,2 & 122,8 & 169,9 & 172,2 & 12,5 & 1069,4 \\
\hline 2007 & 45,1 & 0,0 & 42,4 & 17,5 & 91,4 & 99,7 & 98,2 & 317,2 & 149,0 & 269,3 & 6,0 & 2,5 & 1138,3 \\
\hline 2008 & 0,0 & 5,8 & 11,1 & 43,6 & 174,4 & 61,0 & 85,8 & 174,7 & 210,0 & 235,7 & 87,9 & 0,9 & 1090,9 \\
\hline 2009 & 11,5 & 1,7 & 17,5 & 31,4 & 154,7 & 43,6 & 57,0 & 166,6 & 115,6 & 156,6 & 71,4 & 0,0 & 827,6 \\
\hline 2010 & 1,8 & 4,1 & 0,0 & 151,8 & 330,4 & 371,5 & 213,9 & 201,3 & 145,8 & 75,6 & 188,4 & 13,7 & 1698,3 \\
\hline 2011 & 9,7 & 12,2 & 5,7 & 64,3 & 163,8 & 135,5 & 241,9 & 130,5 & 182,1 & 77,5 & 59,4 & 12,8 & 1095,4 \\
\hline 2012 & 0,0 & 0,0 & 22,4 & 147,0 & 103,5 & 134,0 & 148,1 & 257,1 & 190,1 & 137,8 & 44,1 & 4,3 & 1188,4 \\
\hline 2013 & 0,0 & 0,0 & 33,4 & 125,4 & 99,7 & 88,4 & 105,2 & 315,6 & 149,6 & 153,2 & 130,9 & 6,5 & 1207,9 \\
\hline 2014 & 0,4 & 0,0 & 0,0 & 32,9 & 52,4 & 95,3 & 133,8 & 147,2 & 335,6 & 273,1 & 88,4 & 25,4 & 1184,5 \\
\hline
\end{tabular}

\subsection{Procedimientos aplicados para el control de calidad de datos climáticos}

Se aplicó el control de calidad a las series de precipitación mensual de las estaciones del INIA, mediante la determinación de datos faltantes, el cálculo de estadística descriptiva, análisis de concentración y tendencia de series temporales y análisis de dispersión de acuerdo a la metodología propuesta por Parra y Cortez (2005); y los procedimientos estadísticos desarrollados por Ablan et al. (2008) y Olivares et al. (2013) en estaciones climatológicas a nivel nacional.

La tabla 3 presenta un resumen de los procedimientos estadísticos aplicados a las series de precipitación mensual, el principal problema característico lo constituyeron los datos faltantes mensuales, lo que afectó la longitud de las series que quieren analizarse. El resto de los procedimientos estadísticos arrojó resultados adecuados dentro de lo esperado.

Tabla 3. Resultados de la aplicación de los procedimientos para el control de calidad de las series de precipitación de las estaciones agrometeorológicas durante el período histórico.

\begin{tabular}{cccccc}
\hline Estación & $\begin{array}{c}\text { Datos } \\
\text { Faltantes } \\
\text { Mensuales (\%) }\end{array}$ & Datos Extremos & $\begin{array}{c}\text { Estadística } \\
\text { Descriptiva }\end{array}$ & $\begin{array}{c}\mathbf{N}^{\circ} \text { de series } \\
\text { aleatorias }\end{array}$ & $\begin{array}{c}\text { Análisis de dis- } \\
\text { persión }\end{array}$ \\
\hline CENIAP & 3 & No & N & 11 & N \\
Tapipa - Padrón & 38 & No & N & 12 & N \\
Ocumare de la Costa & 2 & No & N & 11 & N \\
\hline
\end{tabular}

Nota: (N: Patrón Normal esperado) 
Para detectar los valores extremos mensuales en las series de precipitación se usó el criterio basado en la desviación absoluta de la mediana (mad) de acuerdo con Crawley (2002) y Ablan et al. (2008). Para cada estación seleccionada, se calcularon los parámetros estadísticos descriptivos reportados por Olivares et al. (2013): precipitación media mensual, máxima mensual, desviación estándar, coeficiente de variación, percentiles $25 \%, 50 \%, 75 \%$, la asimetría, kurtosis y desviación absoluta de la mediana.

Adicionalmente el análisis de dispersión de las series temporales se realizó a través de la representación gráfica de Boxplots con determinación de valores atípicos moderados y severos, de acuerdo a la metodología propuesta por Parra y Cortez (2005).

\subsection{Determinación del Índice de Precipitación Estandarizado (SPI)}

Una metodología para determinar la magnitud de la sequía debe facilitar el análisis de las relaciones de la sequía con los factores modificadores del clima y demás elementos atmosféricos (Colotti et al., 2013). Frecuentemente se utilizan variables que incluyen la precipitación, evapotranspiración, temperatura, la humedad del suelo y la capacidad de almacenamiento, entre otras; sin embargo, se han desarrollado métodos simplificados que requieren de una sola variable como la precipitación y permiten la cuantificación de la sequía en zonas en las que no se dispone de datos del resto de las variables mencionadas.

En este trabajo, para la caracterización de la sequía en las áreas bajo estudio, se utilizó el Índice de Precipitación Estandarizado (SPI, Standardized Precipitation Index), desarrollado por Mckee et al. (1993). De modo general, para el cálculo se pueden describir dos etapas: la primera consiste en ajustar distribución teórica Gamma a la serie histórica de la precipitación. La segunda etapa consiste en convertir la serie de precipitación ajustada a la distribución Gamma en una distribución normal estandarizada con media 0 y varianza 1 , cuyo resultado representa el índice SPI (Figura 2) (Edwards y Mckee, 1997). 
El análisis de la distribución de la lluvia mediante la distribución gamma generalizada permite la obtención de las estimaciones de los parámetros por máxima verosimilitud, lo que garantiza, en estos casos, un buen ajuste a la distribución empírica.

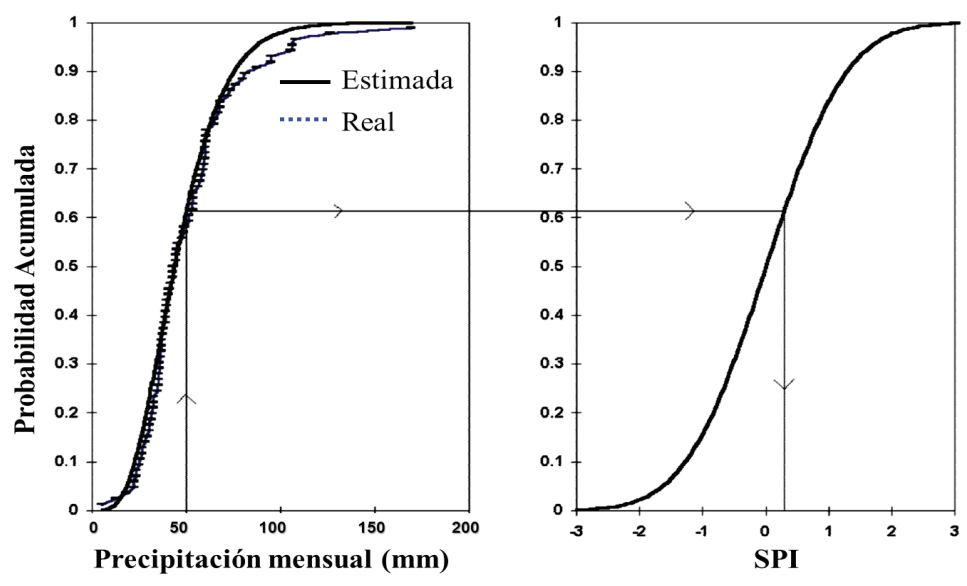

Figura 2. Ejemplo de transformación de una distribución gamma ajustada a una distribución normal estandarizada. Fuente: adaptada de Edwards y McKee, 1997.

De acuerdo a lo reportado por Thom (1958) es recomendable la aplicación de la distribución Gamma, debido en principio a la variabilidad natural de las precipitaciones y a su distribución asimétrica respecto a los valores medios, esta distribución posee ventajas con respecto a la Gaussiana, ya que se adapta a todo tipo de asimetrías, además de estar definida solo para valores positivos, que la hace apta para representar frecuencias de precipitación (Young, 1992; Serio et al., 2010; Colotti et al., 2013; Olivares et al., 2016).

El índice representa el número de desviaciones estándar en que el valor transformado de la precipitación se desvía del promedio histórico, representado por el valor cero. Los valores Negativos del SPI representan déficit de precipitación y aquellos Positivos asociados a datos de precipitación superiores al promedio histórico. 


\subsection{Pasos para el cálculo de SPI}

Los pasos para calcular la magnitud de la sequía se presentan en la figura 3, iniciando con la acumulación de los datos de precipitación mensual con la intención de observar la extensión temporal del fenómeno. En este aspecto, Komuscu (1999) establece que al utilizar una escala temporal mensual, la frecuencia de la sequía se incrementa y su duración disminuye, lo contrario ocurre en escalas mayores, donde la frecuencia de la sequía disminuye pero presenta un efecto más prolongado. La metodología para el cálculo del SPI en series de precipitación de Venezuela se detallan en los estudios desarrollados por Olivares et al. (2016) y Olivares (2017).

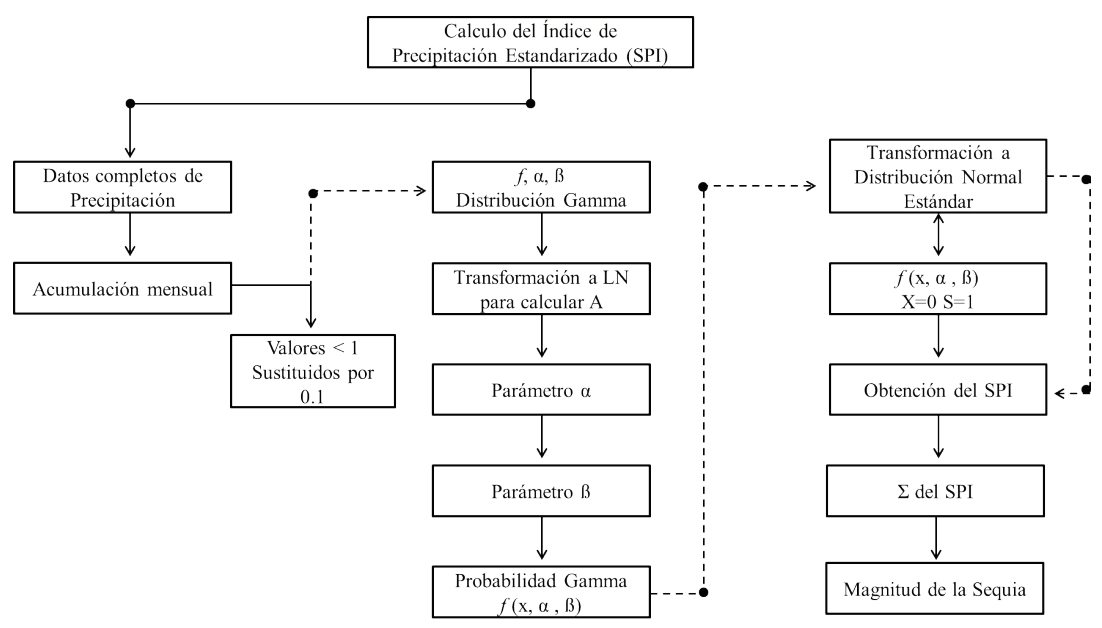

Figura 3. Procedimiento para el cálculo del Índice de Precipitación Estandarizado (SPI). Fuente: adaptado de Colotti et al., 2013.

\subsection{Categorización de la intensidad del SPI}

McKee et al. (1993) utilizaron el sistema de clasificación mostrado en la tabla 4, los valores de SPI que se muestran a continuación. Para definir las distintas intensidades de la sequía según los distintos valores de SPI. Estas categorías están referidas al fenómeno de la sequía meteorológica (aquellas cuyo valor de SPI es negativo) y por tanto, 
corresponden a eventos secos coyunturales, mas no constituyen una condición de aridez (Colotti et al., 2013)

Tabla 4. Clasificación del SPI. Fuente: Adaptado de McKee et al., 1993.

\begin{tabular}{cc}
\hline SPI & Categoría \\
\hline 2,0 y más & extremadamente húmedo \\
1,5 a 1,99 & muy húmedo \\
1,0 a 1,49 & moderadamente húmedo \\
$-0,99$ a 0,99 & normal o aproximadamente normal \\
$-1,0$ a $-1,49$ & moderadamente seco \\
$-1,5$ a $-1,99$ & severamente seco \\
-2 y menos & extremadamente seco \\
\hline
\end{tabular}

\subsection{Determinación de la magnitud, duración y frecuencia de ocurrencia de la sequia}

Para calcular la magnitud del período seco durante un año cualquiera, en una estación determinada, se empleó una variante del método original propuesto por Edwards y Mckee (1997) donde se acumularon los SPI mensuales cuya magnitud era igual o inferior a -1 , y cuando el SPI era mayor a -1 se sustituyó por un cero. Bajo este enfoque, un valor de SPI >-1 indica una condición normal o húmeda (Ecuación 1)

$$
\text { Sí y sólo sí } \mathrm{SPI}_{\mathrm{i}}<0
$$

Dónde (MS): representa la magnitud de la sequía para el período evaluado, (SPI): es el índice SPI para series de lluvia acumulada mensual. La tabla 5 muestra las categorías de la magnitud de la sequía.

Tabla 5. Magnitud de la sequía meteorológica.

\begin{tabular}{cc}
\hline MS & Categoría \\
\hline $0,1-0,9$ & Normal \\
$1-1,99$ & Leve
\end{tabular}


$2-2,99$

3 - 3,99

$4-4,99$
Poco fuerte

Fuerte

Muy fuerte

$>5$

Extremadamente fuerte

Fuente: adaptado de Hernández, 2008.

La frecuencia de ocurrencia se determinó mediante el número de casos de sequía que se producen durante un período determinado, estableciendo así, la probabilidad empírica de que ocurra una sequía de determinada magnitud. Para la realización de todos los cálculos, se utilizó una hoja de cálculo de Microsoft Office Excel 2013.

\section{Resultados}

\subsection{Magnitud de la sequía}

El valor del SPI mensual representa la intensidad de la sequía, es decir, que tan grande es el déficit de precipitación, de acuerdo a la escala temporal utilizada, la cual se calcula sistemáticamente, para cada mes, y no representa necesariamente la gravedad de la totalidad del evento seco, ya que para establecer la gravedad del evento de sequía se requiere del análisis de la magnitud y duración, aunado al de la intensidad.

La figura 4 muestra la intensidad de la sequía de todo el período de registro en la estación CENIAP. De acuerdo con los registros de lluvia de la estación se presentaron valores de sequía extrema (SPI -2 o menos) que representan el 1,89\%, mientras que la intensidad severa (SPI entre-1,99 a -1,5) alcanzó el 2,27\%. La Intensidad predominante es la moderada (SPI entre $-1,49$ a -1) constituida por $6,82 \%$ del total de SPI mensuales evaluados. 

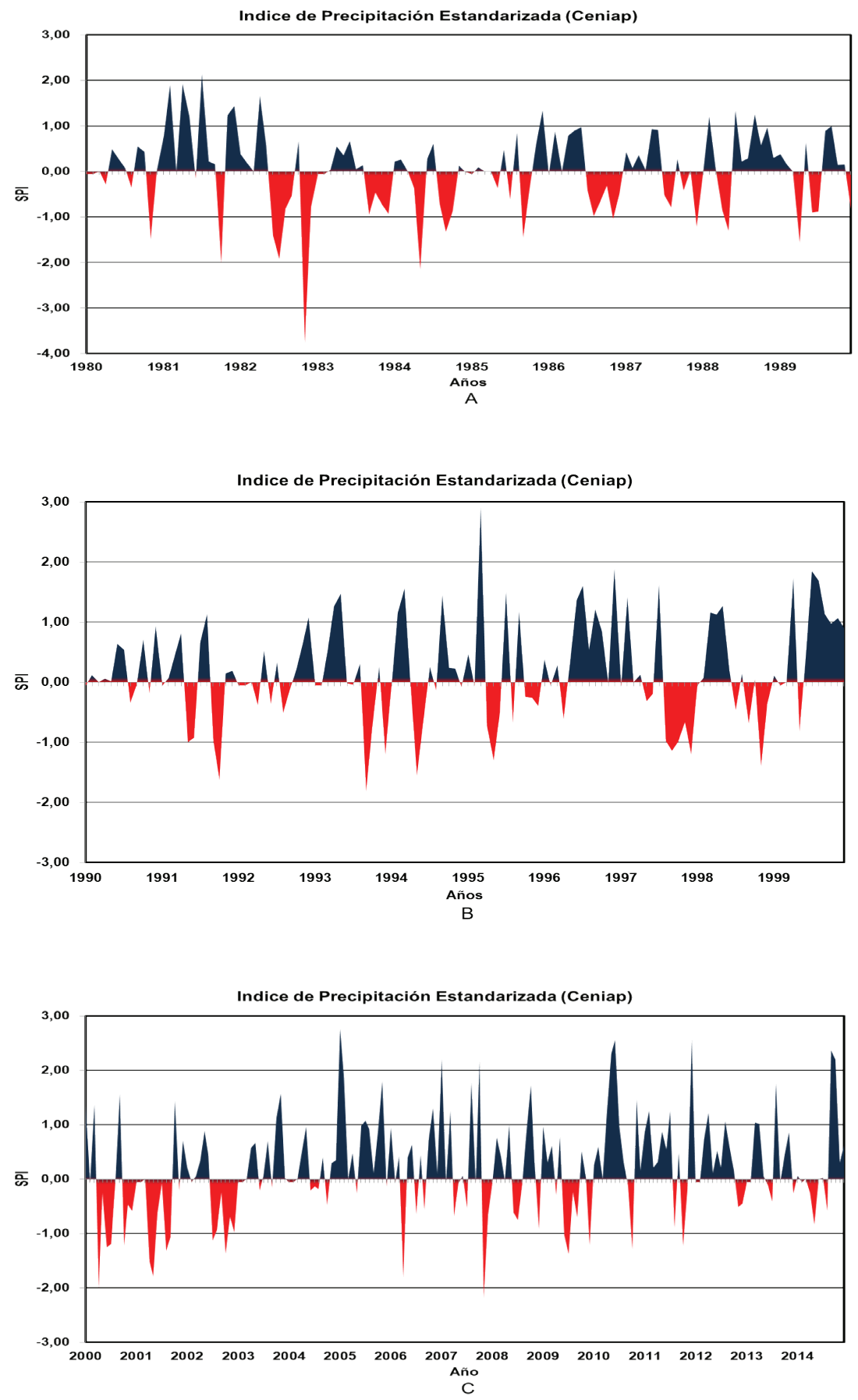

Figura 4. Transcurso del SPI mensual en la estación CENIAP (A. período 1980-1989; B. periodo 1990-1999; C. periodo 2000-2014). 
Los valores de intensidad de la sequía para la estación Tapipa-Padrón se presentan en la figura 5. De acuerdo a los registros de precipitación se presentaron valores de sequía extrema que constituyen el 3,18\%, mientras que la intensidad severa representa el 4,14\%. La intensidad que predomina es la moderada, la cual se presenta en un $7,01 \%$ del total de SPI mensual.

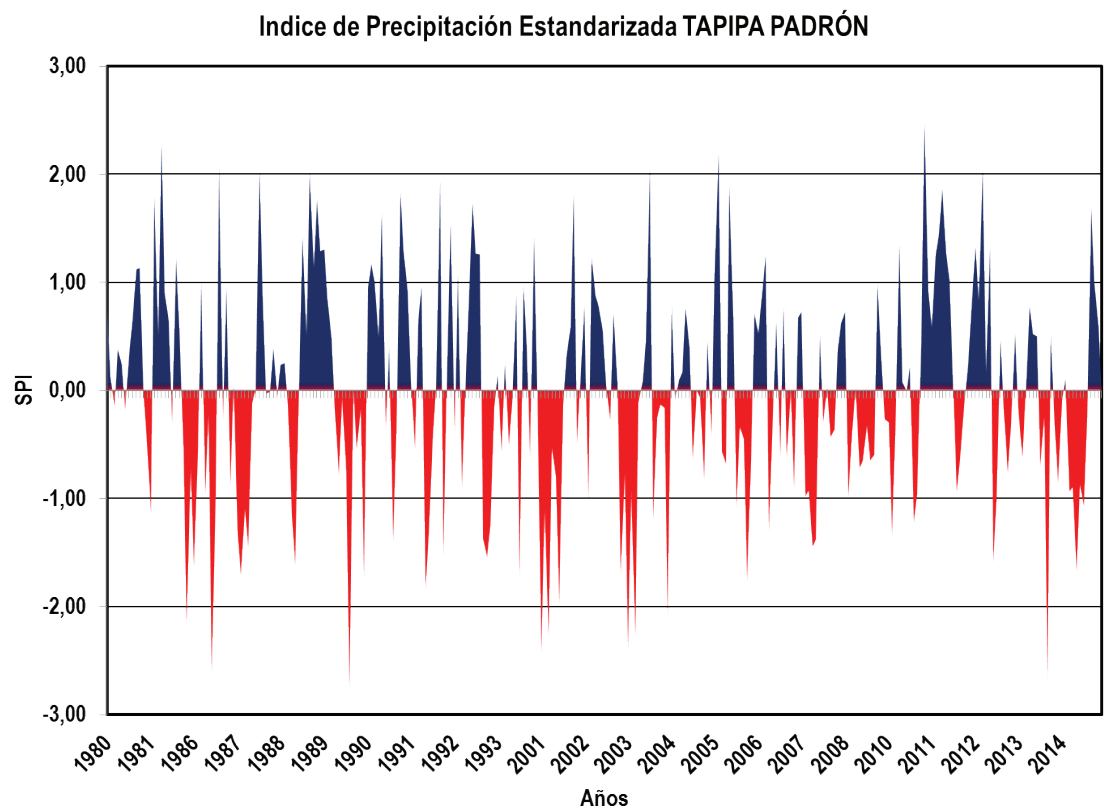

Figura 5. Transcurso del SPI mensual para el periodo (1980-2014) en la estación Tapipa-Padrón.

La figura 6 muestra la intensidad de la sequía de todo el período de registro en la estación Ocumare de la Costa. De acuerdo a los registros de lluvia de la estación se presentaron valores de intensidad severa, los cuales alcanzó el 1,00\%. La Intensidad predominante es la moderada constituida por $11,67 \%$ del total de SPI mensuales evaluados. 


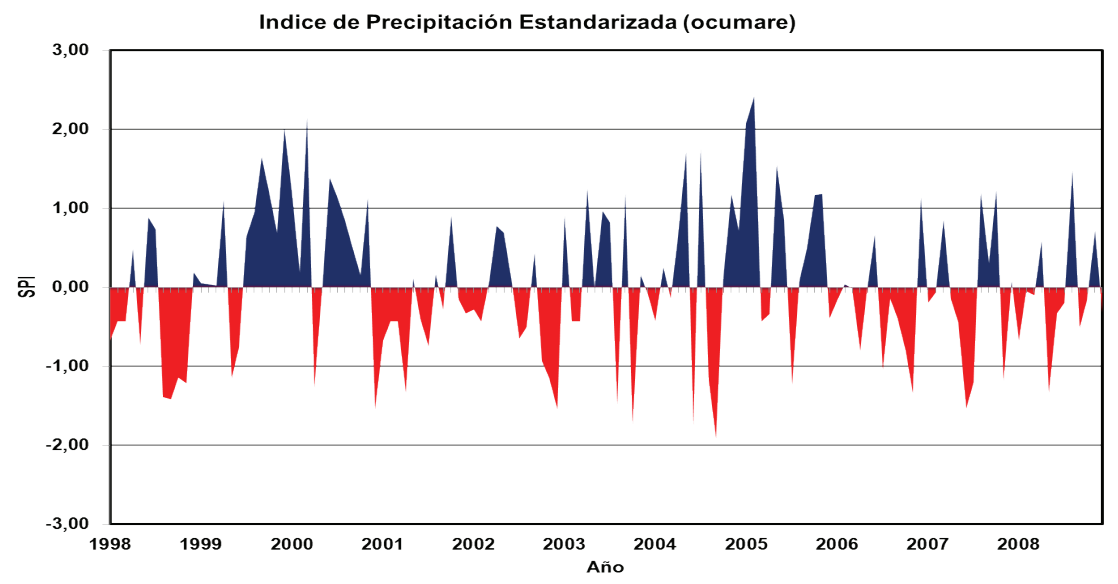

Figura 6. Transcurso del SPI mensual para el periodo (1998-2008) en la estación Ocumare de la Costa.

Venezuela es uno de los países débilmente afectado por el fenómeno El Niño debido a que el sistema climático tiene una menor dependencia del Océano Pacífico. De acuerdo con estudios realizados por CONICIT (1998); Martelo (2000); Cárdenas et al. (2002); Martelo (2003) y Olivares (2017), se puede afirmar que los eventos El Niño en el país están relacionados con situaciones de sequía e incremento de la temperatura. Se podría establecer que sobre el territorio nacional se mezclan situaciones de influencia de la Zona de Convergencia Intertropical del Atlántico y del Pacífico, combinaciones de vaguadas en altura, y restos de frentes fríos de origen Sur y Norte. Dado que El Niño tiene su efecto entre diciembre y abril, que corresponde con la época seca en el país, esta se hace más cálida y árida que en condiciones normales, afectando el inicio de la época lluviosa.

\subsection{Magnitud de la sequía}

Para describir la gravedad de los eventos de sequía según el SPI mensual, se calculó la magnitud de cada una de las sequías detectadas; clasificadas en cinco clases anteriormente señaladas.

De acuerdo con los resultados de magnitud, predominan las sequías poco fuertes en las estaciones CENIAP (Figura 7a), seguidas de 
las extremadamente fuertes en las estaciones Tapipa-Padrón (Figura 7b). Como la Magnitud depende tanto de la duración como de la intensidad, en general las sequías de magnitud leve y poco fuerte están asociadas a duraciones de una agrupación temporal (solo un mes), aunque puedan tener una intensidad alta.

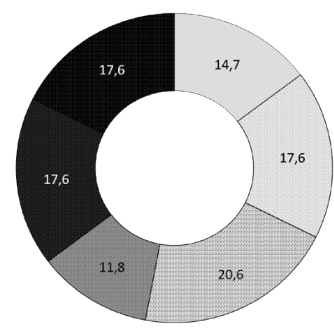

(A)

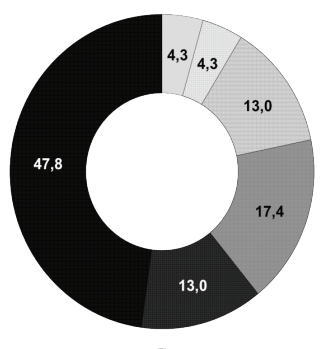

(b)

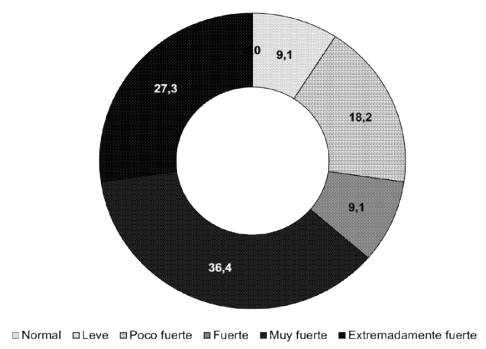

(c)

Figura 7. Frecuencia de ocurrencia de eventos de sequía según las clases de magnitudes (SPI mensual) en el período de estudio: (a) Estación CENIAP; (b) Estación Tapipa Padrón; (c) Estación Ocumare de la Costa.

Como caso típico, es común que hayan estaciones que presentan alto riesgo de ocurrencia, pero son poco vulnerables por no poseer actividades agrícolas importantes (Estación CENIAP), los recursos financieros para prevenir o mitigar la sequía podrían dirigirse hacia otra zona con menor riesgo de ocurrencia, pero más vulnerable por desarrollar sistemas de producción agrícola intensiva o por poseer ciertos recursos hídricos que obligatoriamente tienen que protegerse (Estación Tapipa-Padrón). También una posible estrategia puede estar 
ligada a los planificadores ambientales, quienes podrían establecer el carácter prioritario del desarrollo sostenible de los pequeños productores agrícolas rurales (cercanos a las estaciones Tapipa-Padrón), en términos de seguridad agroalimentaria endógena, cuyo riesgo a sequias extremadamente fuertes es importante de considerar.

A título ilustrativo, la distribución de las magnitudes de las sequías en las estaciones CENIAP y Tapipa-Padrón se representan en la figura 8 , siendo la estación de Tapipa-Padrón la que mayor número de magnitudes extremadamente fuertes (11 años) en comparación con la estación CENIAP cuyas magnitudes ubicadas en esta categoría están alrededor de 6 años. El evento de sequía ocurrido desde inicios del periodo pre-húmedo (abril) del 2001 a inicios (febrero y marzo) de 2002, constituye uno de los más intensos que se haya conocido en la mayoría del territorio nacional a lo largo del registro histórico.

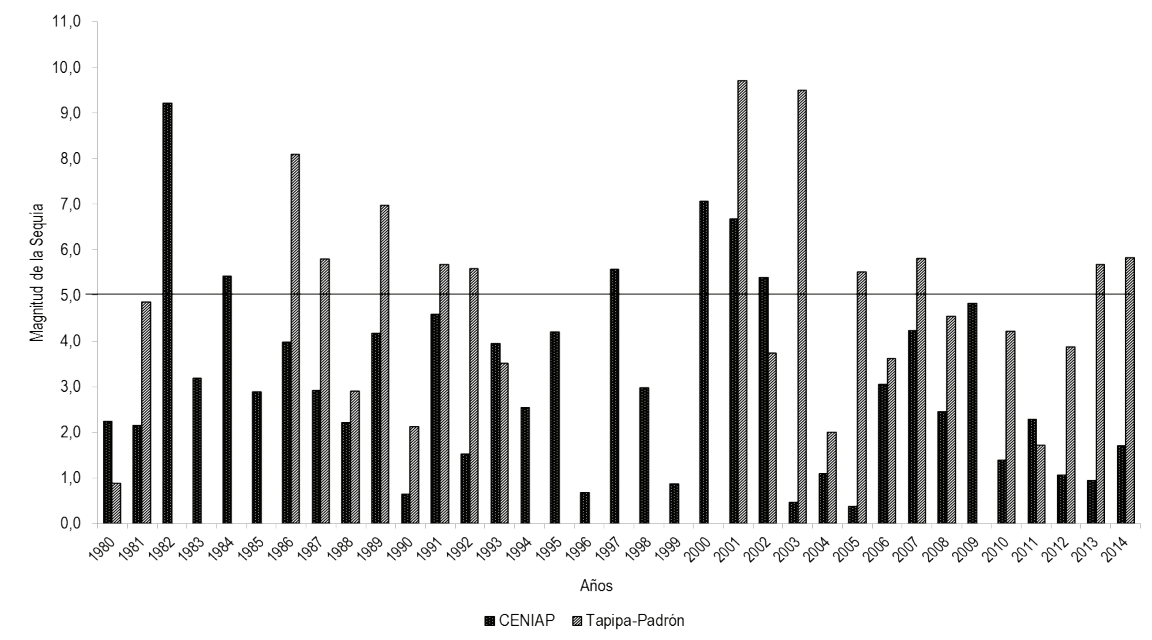

Figura 8. Distribución de las magnitudes de las sequías durante el periodo (1980-2014) para las estaciones CENIAP y Tapipa-Padrón.

\subsection{Duración de las magnitudes máximas de las sequías}

Los resultados anteriormente discutidos evidenciaron que en algunas estaciones bajo estudio se han presentado ciertos eventos de magnitud extremadamente fuertes; sin embargo, estos episodios han sido localizados. En la tabla 6 se presentan las siete magnitudes máxi- 
mas de las sequías detectadas en la zona de estudio, para el grupo de estaciones de estudio.

En la estación CENIAP, la mayor magnitud fue de 9,20, ubicándose en dos meses lluviosos del año 1982. También para el año 2001, hubo una disminución en el total anual de lluvia registrada en la zona asociada al evento de sequía durante dos meses del pre húmedo y dos meses del periodo lluvioso. Este mismo comportamiento se presentó en la estación Tapipa-Padrón para ese mismo año asociado a la sequía de magnitud 9,70 y de tres meses consecutivos de duración.

Tabla 6. Duración de las magnitudes máximas detectadas en las estaciones bajo estudio.

\begin{tabular}{|c|c|c|c|c|}
\hline \multirow{2}{*}{ Estación } & \multirow{2}{*}{ Magnitud } & \multicolumn{2}{|c|}{ Duración } & \multirow{2}{*}{$\begin{array}{l}\text { Lluvia total anual } \\
\qquad(\mathrm{mm})\end{array}$} \\
\hline & & Periodo & Año & \\
\hline \multirow{3}{*}{ CENIAP } & 9,20 & Jun-Jul/Nov & 1982 & 842,3 \\
\hline & 7,06 & Abr/Jun-Jul/Oct & 2000 & 816,2 \\
\hline & 6,68 & Abr-May/Ago-Sep & 2001 & 723,1 \\
\hline \multirow{3}{*}{ Tapipa-Padrón } & 9,70 & Ene-Mar/Jun & 2001 & 2083,4 \\
\hline & $\mathbf{9 , 5 0}$ & Ene/Mar/Ago/Dic & 2003 & 2178,7 \\
\hline & 8,09 & Ene/Jun & 1986 & 2170,3 \\
\hline Ocumare de la Costa & 7,43 & Ago-Nov & 1998 & 578,4 \\
\hline
\end{tabular}

\subsection{Discusión de los resultados}

Según Hernández (2015), la sequía ocurre cuando las precipitaciones son inferiores al promedio histórico registrado en un lugar ó región determinada y dependerá de la duración y la superficie que abarque. En los últimos cuarenta años, recordando 1973-74, 1982-83, 1997-98, 2002-2003, 2009-2010, 2013-2014 y recientemente mayo 2015, se constituyó en una amenaza climática afectando grandes porciones del territorio nacional que generó a la población en general pérdidas económicas, sociales y culturales sobre los sectores agroalimentarios, energéticos, hídricos, turismo, reduciendo e inclusive perdiendo la biodiversidad (incendios forestales), entre otros.

En el contexto de la agricultura, la sequía no comienza cuando cesa la lluvia, sino cuando las raíces de las plantas no pueden obtener 
humedad del suelo, aunque en este estudio no se hace un análisis exhaustivo de la sequía agrícola, se generan inferencias de lo que puede ocurrir cuando la cantidad de precipitación y su distribución, las reservas de agua del suelo y las perdidas por evaporación se combinan para causar considerables disminuciones en el rendimiento del cultivo.

Ante este panorama, el MARN (2004) plantea la necesidad de prevenir y combatir la desertificación, siendo prioritario emprender acciones de forma multidisciplinaria e interinstitucional, teniendo como protagonista más importante a la comunidad y donde se incorpore el elemento del género como agente principal en esta lucha contra la desertificación y por la mitigación de la sequía.

\section{Conclusiones}

De acuerdo a los resultados obtenidos, durante los períodos 20022003, 2009-2010, 2013-2014, las precipitaciones registradas fueron originadas por el pasaje de las ondas tropicales y en segundo orden por una ligera actividad de la Zona de Convergencia Intertropical (ZCIT), sin embargo, las lluvias no alcanzaron valores significativos, ubicándose en gran parte del territorio nacional por debajo de los promedios históricos registrados, lo que provocó anomalías negativas de precipitaciones; es decir, déficit acumulado de precipitación desde abril a septiembre esos años, que produjeron sequías y afectaron los niveles de algunos embalses en la zona central del país y la actividad agrícola.

El estudio de la sequía desarrollado en este trabajo de investigación, constituye la columna vertebral para la planificación del espacio debido esencialmente a que la generación de este tipo de datos aquí reportados, sirve para que los organismos pertinentes consideren ciertas medidas preventivas y mitigantes. De acuerdo a los valores de SPI reportados en las regiones bajo estudios existe el fenó- 
meno de la sequía meteorológica, mostrando variaciones espaciales y temporales en la intensidad, magnitud, duración y frecuencia de ocurrencia.

Este trabajo es una herramienta muy útil para los Planificadores ambientales, que les permitirá reforzar el diagnóstico de la vulnerabilidad a la sequía y la creación de capacidad institucional sobre este tema, así mismo, la investigación representa el marco de referencia para incluir el impacto del Cambio Climático sobre la misma.

\section{Referencias}

Ablan, M., Andressen, R., Vargas, M. P. \& Acevedo, M. (2008). Propuesta metodológica para el control de calidad de datos de precipitación. Agronomía Trop, 58 (1), 57-60. Recuperado de http://sian.inia.gob.ve/repositorio/revistas_ci/Agronomia\%20 Tropical/at5801/pdf/ablan_m.pdf

Campos, D. (2005). Agroclimatología cuantitativa de cultivos. Editorial Trillas. México, D. F. 320p.

Cárdenas, P., García, L. F. \& Gil, A. (2002). Impacto de los eventos El Niño - Oscilación del Sur en Venezuela. Caracas: Corporación Andina de Fomento. Recuperado de: http://www.inameh. gob.ve/documentos/Impacto_ENOS_ParteI.pdf

Colotti, E., Cedeño, M. \& Montañez, C. (2013). La sequía meteorológica y la variación de la superficie agrícola en la Isla de Margarita, estado Nueva Esparta, Venezuela período 1972-2004. Terra Nueva Etapa, XXIX (45), 11-53. Recuperado de http:// www.redalyc.org/pdf/721/72130180002.pdf

Consejo Venezolano de Investigaciones Científicas y Tecnológicas. CONICIT. (1998). El fenómeno El Niño y su posible influencia sobre el territorio de Venezuela. Caracas: CNMeH. 25p. 
Crawley, M. (2002). Statistical Computing: An Introduction to Data Analysis using S-plus. John Wiley \& Sons, England. 761 p.

Edwards, D. C. \& McKee, T.B. (1997). Characteristics of 20th Century drought in the United States at multiple time scales. Atmospheric Science Paper No. 634. Climatology Report (97-2), USA: Colorado State University, Fort. Collins.

Grupo Intergubernamental de Expertos sobre el Cambio Climático. IPCC. (2013). Cambio climático 2013: bases físicas. Resumen para responsables de politicas. Ginebra, Suiza: OMM/ PNUMA. 27p.

Hernández, R. (2008). Caracterización de la sequía meteorológica en los climas Árido, Semiárido y Subhúmedo seco en los Llanos Centro Orientales de Venezuela, para el manejo de los recursos hídricos. Trabajo de Grado. Centro de Investigación y Postgrado. UNEFA-CIP. Universidad Nacional Experimental Politécnica de la Fuerza Armada, Maracay, Venezuela.

Hernández, R. (2015). Caracterización espacial de la sequía meteorológica (SPI) a nivel semestral noviembre 2014 hasta septiembre 2015, para el territorio nacional. Baruta: INAMEH

Martelo, M. T. (2000). Estudio sobre la posible influencia del fenómeno El Niño - Oscilación del sur (ENOS) sobre el clima de los llanos de Venezuela. En: OMM (Ed.), Reunión de expertos de las asociaciones regionales AR-III y AR-IV sobre fenómenos adversos (pp. 111-118). Ginebra: OMM

Martelo, M. T. (2003). Influencia de las variables macroclimáticas en el clima de Venezuela. Caracas: Ministerio del Ambiente y los Recursos Naturales, Dirección General de Cuencas Hidrográficas, Dirección de Hidrología, Meteorología y Oceanía. 72p.

Mckee, T., Doesken, N., \& Kleist, J. (1993). The relationship of drought frequency and duration to time scales. En: Proceedings of the Eighth Conference on Applied Climatology, 17-22 
January 1993, Anaheim, California, USA. American Meteorological Society.

Ministerio de Ambiente y de los Recursos Naturales. MARN. (2004).

Programa de acción nacional de lucha contra la desertificación y mitigación de la sequía de la República Bolivariana de Venezuela. Caracas: Editorial Fundambiente. 106p. Recuperado de http://www.unccd.int/ActionProgrammes/venezuela-spa2004.pdf

Olivares, B. (2017). La sequía meteorológica en territorios agrícolas de Venezuela: un análisis temporal del fenómeno meteorológico y su impacto en la agricultura venezolana. Saarbrücken, Alemania, Editorial Académica Española. 145 p.

Olivares, B., Cortez, A., Rodríguez, M., Parra, R., Lobo, D. y Rey, J.C. (2016). Análisis temporal de la sequía meteorológica en localidades semiáridas de Venezuela. UGCiencia 22 (1):1124.

Olivares, B., Cortez, A., Parra, R., Rodríguez, M. \& Guevara, E. (2013). Aplicación de procedimientos estadísticos para el control de calidad de las series de precipitación mensual de los llanos orientales venezolanos. Revista de la Facultad de Agronomía, 30 (3): 367-391.

Organización Meteorológica Mundial. OMM. (2006). Vigilancia y alerta temprana de la sequía: conceptos, progresos y desafíos futuros. Información meteorológica y climática para el desa-

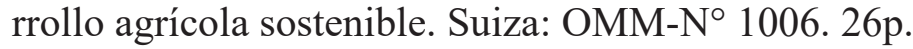

Parra, R. \& Cortez, A. (2005). Control de calidad de series de precipitación de las series de precipitación del INIA Venezuela en el periodo 1970-2000. Rev. Arg. de Agrometeorología, (5-6): 63-73.

Serio, L., Martin, P. \& Murphy, G. (2010). Evaluación de una metodología de pronóstico estadístico para la condición hídrica del 
suelo en la región pampeana argentina. Agriscientia, XXVII, 11-17.

Solano, O.J., Vázquez, R.J., Centella, A. \& Lapinel, B.P. (2007). Una aproximación al Conocimiento de la Sequía en Cuba y sus efectos en la Producción Agropecuaria. Zonas Áridas 11(1): $100-85$.

Thom, H. (1971). Some methods of climatological analysis. Nota técnica No 81, OMM No 199, TP 103, Secretaría de la OMM, Ginebra, Suiza, pp. 1-11.

Wu H., Hayes M.L., Wilhite D.A. \& Svoboda, M.D. (2005). The effect of the length of record on the Standardized Precipitation Index calculation. Int. J. Climato, 25, 505-520.

Young, K. (1992). A three-way model for interpolating for monthly precipitation values. Mon. Wea. Rev, 120, 2561-2569. 